PROCEEDINGS OF THE

AMERICAN MATHEMATICAL SOCIETY

Volume 139, Number 8, August 2011, Pages 2767-2775

S 0002-9939(2011)10704-4

Article electronically published on January 6, 2011

\title{
HAUSDORFF DIMENSION OF THE LIMIT SET OF CONFORMAL ITERATED FUNCTION SYSTEMS WITH OVERLAPS
}

\author{
EUGEN MIHAILESCU AND MARIUSZ URBAŃSKI
}

(Communicated by Bryna Kra)

\begin{abstract}
We give a new approach to the study of conformal iterated function systems with arbitrary overlaps. We provide lower and upper estimates for the Hausdorff dimension of the limit sets of such systems; these are expressed in terms of the topological pressure and the function $d$, counting overlaps. In the case when the function $d$ is constant, we get an exact formula for the Hausdorff dimension. We also prove that in certain cases this formula holds if and only if the function $d$ is constant.
\end{abstract}

\section{INTRODUCTION}

The geometry of limit sets of conformal iterated function systems satisfying the open set condition, that is, with no overlaps, is fairly well understood in the case of a finite alphabet as well as an infinite one; see 2 and the references therein. In particular, the classical version of Bowen's formula holds, identifying, in the case of a finite alphabet, the Hausdorff dimension of the limit set as the unique zero of the pressure function.

It is however a notoriously difficult task to find a formula, or at least to get some good estimates for the Hausdorff dimension of the limit set of a conformal iterated function system with overlaps. All attempts known to us aimed to neutralize the effects of overlaps and to get the classical form of Bowen's formula. The most successful of them was the one based on the concept of transversality (see [8, [7]) when the results were only generic, holding for almost all members of parametrized families of iterated function systems.

Our approach in this paper is drastically different. Firstly, we deal with one fixed conformal iterated function system having arbitrary overlaps. Secondly, we fully acknowledge the existence of overlaps and recognize their influence on the value of the Hausdorff dimension of the limit set. We get two estimates, namely lower and upper bounds in Theorem 3.1 and Theorem 4.1, both quantitatively incorporating

Received by the editors February 9, 2010 and, in revised form, July 17, 2010.

2010 Mathematics Subject Classification. Primary 37C45, 37D35, 28A80.

Key words and phrases. Conformal iterated function systems, Hausdorff dimension, overlaps, equilibrium states.

The research of the first author supported by CNCSIS-UEFISCSU through project PN II IDEI-1191/2008.

The research of the second author supported in part by NSF grant DMS 0700831.

(C)2011 American Mathematical Society Reverts to public domain 28 years from publication 
overlaps. In the case when the function $d$, counting overlaps, is constant, we get an exact formula (Corollary 4.2) for the Hausdorff dimension.

Corollary 4.2 also says that this formula holds if and only if the function $d$ is constant. We would like to add that in the case of a smooth dynamical system $f: M \rightarrow M$, where $M$ is a smooth Riemannian manifold, we obtained somewhat analogous estimates for the stable dimension on a hyperbolic basic set $\Lambda \subset M$; see [5] and [6]. Also in [4, one of us studied the dynamics of a class of skew products with overlaps in fibers.

\section{IFS PRELIMINARIES}

Fix an integer $q \geq 1$ and a real number $s \in(0,1)$. Let $X$ be a compact subset of $\mathbb{R}^{q}$ such that $X=\overline{\operatorname{Int} X}$. Suppose that $V$ is a bounded connected open subset of $\mathbb{R}^{q}$ such that $X \subset V$.

Also fix an arbitrary finite set $E$ called in the sequel an alphabet. A system $\mathcal{S}=\left\{\phi_{e}: V \rightarrow V\right\}_{e \in E}$ of $C^{1+\varepsilon}$ conformal injective maps from $V$ to $V$ is called a conformal iterated function system if $\phi_{e}(X) \subset X$ for all $e \in E$ and $\left\|\phi_{e}^{\prime}\right\|=$ $\sup \left\{\left|\phi_{e}^{\prime}(x)\right|: x \in V\right\} \leq s<1$ for all $e \in E$. Here, $\phi_{e}^{\prime}(x): \mathbb{R}^{q} \rightarrow \mathbb{R}^{q}$ is the derivative of the map $\phi_{e}: V \rightarrow V$ evaluated at the point $x$; it is a similarity map, and $\left|\phi_{e}^{\prime}(x)\right|$ is its operator norm or, equivalently, its scaling factor.

Note that we do not assume any version of the open set condition, i.e. allow any overlaps of the sets $\phi_{a}(X)$ and $\phi_{b}(X)$, where $a, b \in E$ with $a \neq b$. Let

$$
E^{*}=\bigcup_{n=0}^{\infty} E^{n} \text { and } E^{\infty}=\left\{\left(\omega_{n}\right)_{n=1}^{\infty}: \forall_{n \geq 1} \omega_{n} \in E\right\} .
$$

If $\tau \in E^{\infty}$ and $n \geq 0$, we put $\left.\tau\right|_{n}=\tau_{1} \ldots \tau_{n}$. Now fix $\omega \in E^{\infty}$ and notice that $\left(\phi_{\left.\omega\right|_{n}}(X)\right)_{n=1}^{\infty}$ is a descending sequence of compact sets such that $\operatorname{diam}\left(\phi_{\left.\omega\right|_{n}}(X)\right) \leq$ $\tilde{D} s^{n} \operatorname{diam}(X)$, where the number $\tilde{D} \geq 1$ is due to the fact that we do not assume the set $X$ to be convex. Therefore, the intersection $\bigcap_{n=1}^{\infty} \phi_{\left.\omega\right|_{n}}(X)$ is a singleton, and we denote its only element by $\pi(\omega)$. So, we have defined a map $\pi: E^{\infty} \rightarrow X$ which is Lipschitz continuous if $E^{\infty}$ is endowed with the metric $d_{s}(\omega, \tau)=s^{|\omega \wedge \tau|}$, where $\omega \wedge \tau$ is the longest common initial block of $\omega$ and $\tau$; we also set $s^{\infty}=0$.

The limit set (or the attractor) $J=J_{\mathcal{S}}$ of the system $\mathcal{S}$ is, by definition, equal to $\pi\left(E^{\infty}\right)$. Clearly, we have

$$
J_{\mathcal{S}}=\bigcap_{n=1}^{\infty} \bigcup_{|\omega|=n} \phi_{\omega}(X)
$$

and $J_{\mathcal{S}}$ is the unique compact set contained in $X$ satisfying the following selfconformality condition

$$
J_{\mathcal{S}}=\bigcup_{e \in E} \phi_{e}\left(J_{\mathcal{S}}\right)
$$

and, by induction,

$$
J_{\mathcal{S}}=\bigcup_{|\omega|=n} \phi_{\omega}\left(J_{\mathcal{S}}\right), \quad n \geq 1
$$

Let $\sigma: E^{\infty} \rightarrow E^{\infty}$ be the (one-sided) shift map, i.e. $\sigma\left(\left(\omega_{n}\right)_{n=1}^{\infty}\right)=\left(\left(\omega_{n+1}\right)_{n=1}^{\infty}\right)$. Let $\psi: E^{\infty} \rightarrow \mathbb{R}$ be the function defined by the formula

$$
\psi(\omega)=\log \left|\phi_{\omega_{1}}^{\prime}(\pi(\sigma(\omega)))\right|, \quad \omega \in E^{\infty} .
$$


As all the maps $\phi_{e}, e \in E$, are $C^{1+\varepsilon}$ and $\left\|\phi_{e}^{\prime}\right\| \leq s<1$ for all $e \in E$, and since the alphabet $E$ is finite, one can easily prove the following two lemmas.

Lemma 2.1. The function $\psi: E^{\infty} \rightarrow \mathbb{R}$ is Hölder continuous.

Lemma 2.2. If $g: E^{\infty} \rightarrow \mathbb{R}$ is Hölder continuous, then there exists a constant $C_{g}>0$ such that

$$
\left|\sum_{j=0}^{n-1} g\left(\sigma^{j}(\omega)\right)-\sum_{j=0}^{n-1} g\left(\sigma^{j}(\tau)\right)\right| \leq C_{g},
$$

for all $n \geq 1$ and all $\omega, \tau \in E^{\infty}$ such that $\left.\omega\right|_{n}=\left.\tau\right|_{n}$.

Now, let us define a function $d: J \rightarrow \mathbb{N}$ by the formula

$$
d(x)=\#\left\{e \in E: x \in \phi_{e}(J)\right\} .
$$

Immediately from this definition we get the trivial, but very useful, formula

$$
\sum_{e \in E: x \in \phi_{e}(J)} d^{-1}(x)=1
$$

for all $x \in J$.

Now let $\kappa: E^{\infty} \rightarrow[1,+\infty)$ be a Hölder continuous function and, for an arbitrary parameter $t \in \mathbb{R}$, consider the potentials $\psi_{\kappa, t}: E^{\infty} \rightarrow \mathbb{R}$ defined as follows:

$$
\psi_{\kappa, t}(\omega)=t \psi(\omega)-\log \kappa(\omega)=t \log \left|\phi_{\omega_{1}}^{\prime}(\pi(\sigma(\omega)))\right|-\log \kappa(\omega), \omega \in E^{\infty} .
$$

One can check easily that $\psi_{\kappa, t}$ is Hölder continuous by using Lemma 2.1 and the Hölder continuity of $\kappa$.

Let $\mathrm{P}(t):=\mathrm{P}\left(\psi_{\kappa, t}\right)$ be the topological pressure of the potential $\psi_{\kappa, t}$ with respect to the dynamical system $\sigma: E^{\infty} \rightarrow E^{\infty}$. Since $\log \left|\phi_{\omega_{1}}^{\prime}(\pi(\sigma(\omega)))\right| \leq \log s<0$, there exists a unique $h_{\kappa} \in \mathbb{R}$ such that $\mathrm{P}\left(\psi_{\kappa, h_{\kappa}}\right)=0$. Let $\tilde{\mu}_{t}$ be the unique shift-invariant Gibbs (equilibrium) state of the Hölder continuous potential $\psi_{\kappa, t}: E^{\infty} \rightarrow \mathbb{R}$, and let

$$
\mu_{t}=\tilde{\mu}_{t} \circ \pi^{-1} .
$$

Clearly, $\mu_{t}(J)=1$. For every $\omega \in E^{*}$, say $\omega \in E^{n}$, let

$$
[\omega]=\left\{\tau \in E^{\infty}:\left.\tau\right|_{n}=\omega\right\} .
$$

This set is called the (initial) cylinder generated by $\omega$. The Gibbs property means that

$$
\tilde{\mu}_{t}\left(\left[\left.\omega\right|_{n}\right]\right) \asymp e^{-\mathrm{P}(t) n}\left\|\phi_{\left.\omega\right|_{n}}^{\prime}\right\|^{t} \prod_{j=0}^{n-1} \kappa^{-1}\left(\pi\left(\sigma^{j}(\omega)\right)\right) .
$$

If $A$ is an arbitrary Borel subset of $J$ and $\mathcal{F} \subset E^{*}$ is a family of mutually incomparable words such that $\pi^{-1}(A) \subset \bigcup_{\omega \in \mathcal{F}}[\omega]$, then

$$
\mu_{t}(A) \leq \sum_{\omega \in \mathcal{F}} \tilde{\mu}_{t}([\omega]) .
$$




\section{LOWER BOUND}

In this section we shall prove the following:

Theorem 3.1. If $\mathcal{S}=\left\{\phi_{e}\right\}_{e \in E}$ is a conformal iterated function system and $\hat{\kappa}$ : $J \rightarrow[1,+\infty)$ is a continuous function such that $d(x) \leq \hat{\kappa}(x)$ for all $x \in J$, then $\mathrm{HD}(J) \geq h_{\kappa}$, where $\kappa=\hat{\kappa} \circ \pi: E^{\infty} \rightarrow \mathbb{R}$.

Proof. Since every real-valued continuous function can be approximated uniformly from above by Hölder (even Lipschitz) continuous functions and since the pressure function is Lipschitz continuous with the Lipschitz constant 1, we may assume without loss of generality that the function $\hat{\kappa}: J \rightarrow[1,+\infty)$ is Hölder continuous. Since $\operatorname{HD}(J) \geq 0$ we may also assume without loss of generality that $h_{\kappa}>0$. Then fix an arbitrary $t \in\left(0, h_{\kappa}\right)$. So, $\mathrm{P}(t)>0$. Since the function $\hat{\kappa}^{-1}: J \rightarrow(0,1]$ is uniformly countinuous, there exists $\eta>0$ so small that

$$
\hat{\kappa}^{-1}(y) \leq e^{\mathrm{P}(t)} \hat{\kappa}^{-1}(x),
$$

for all $x, y \in J$ with $\|y-x\|<\eta$. Since the alphabet $E$ is finite, for every $z \in J$ there exists $R(z) \in(0, \eta)$ such that if $B(z, R(z)) \cap \phi_{e}(J) \neq \emptyset$, then $z \in \phi_{e}(J)$. Consider the open cover $\{B(z, R(z) / 2)\}_{z \in J}$ of the set $J$. Since $J$ is compact, there exists a finite set $F \subset J$ such that

$$
J \subset \bigcup_{z \in F} B(z, R(z) / 2)
$$

Now fix $x \in J$ and

$$
0<r<R_{*}:=\frac{1}{5} \min \{\operatorname{diam}(J), R(\cdot)\} .
$$

By (3.1) there exists $z_{x} \in F$ such that $x \in B\left(z_{x}, R\left(z_{x}\right) / 2\right)$.

We say in the sequel that two words from $E^{*}$ are mutually incomparable if neither is an extension of the other.

Now given a set $B \subset B(x, r)$, we say that a family $\mathcal{F} \subset E^{*}$ consisting of mutually incomparable words is properly placed with respect to the triple $(x, B, r)$ if for all $\omega \in \mathcal{F}$ we have that

$$
B \cap \phi_{\omega}(J) \neq \emptyset .
$$

Immediately from this definition, the definition of $R$ and the restriction on $r>0$, we get that

$$
z_{x} \in \phi_{\omega_{1}}(J)
$$

for all $\omega \in \mathcal{F}$.

Now fix an arbitrary $\tau \in E^{\infty}$ and a family $\mathcal{F} \subset E^{*}$ which is properly placed with respect to $(x, B, r)$ for some $B \subset B(x, r)$. We then have

$$
\begin{aligned}
\Sigma(\mathcal{F}) & :=\sum_{\omega \in \mathcal{F}} e^{-\mathrm{P}(t)|\omega|} \kappa^{-1}(\omega \tau) \kappa^{-1}(\sigma(\omega \tau)) \ldots \kappa^{-1}\left(\sigma^{|\omega|-1}(\omega \tau)\right) \\
& \leq \sum_{\omega \in \mathcal{F}} e^{-\mathrm{P}(t)|\omega|} e^{\mathrm{P}(t)} \hat{\kappa}^{-1}\left(z_{x}\right) \kappa^{-1}(\sigma(\omega \tau)) \ldots \kappa^{-1}\left(\sigma^{|\omega|-1}(\omega \tau)\right) \\
& \leq \sum_{\omega \in \mathcal{F}} e^{-\mathrm{P}(t)(|\omega|-1)} d^{-1}\left(z_{x}\right) \kappa^{-1}(\sigma(\omega \tau)) \ldots \kappa^{-1}\left(\sigma^{|\omega|-1}(\omega \tau)\right) \\
& =\sum_{e \in \mathcal{F}_{1}} d^{-1}\left(z_{x}\right) \cdot \sum_{\omega \in \mathcal{F}(e)} e^{-\mathrm{P}(t)|\omega|} \kappa^{-1}(\omega \tau) \kappa^{-1}(\sigma(\omega \tau)) \ldots \kappa^{-1}\left(\sigma^{|\omega|-1}(\omega \tau)\right),
\end{aligned}
$$


where

$$
\mathcal{F}_{1}:=\left\{\omega_{1} \in E: \omega \in \mathcal{F}\right\} \subset\left\{e \in E: z_{x} \in \phi_{e}(J)\right\},
$$

and then for all $e \in \mathcal{F}_{1}$,

$$
\mathcal{F}(e):=\left\{\omega \in E^{*}: e \omega \in \mathcal{F}\right\}
$$

Notice that for each $e \in \mathcal{F}_{1}$, the family $\mathcal{F}(e)$ consists of mutually incomparable words and $\phi_{e}^{-1}\left(z_{x}\right) \in J$. If $\omega \in \mathcal{F}(e)$, then we have

$$
\emptyset \neq \phi_{e}^{-1}\left(\phi_{e \omega}(J) \cap B\right)=\phi_{\omega}(J) \cap \phi_{e}^{-1}(B)
$$

and

$$
\phi_{e}^{-1}(B) \subset B\left(\phi_{e}^{-1}\left(x_{e}\right), 2 K r\left\|\phi_{e}^{\prime}\right\|^{-1}\right)
$$

where $x_{e}$ is an arbitrary point in $\phi_{e}(J) \cap B$, independent of $\omega$ and $K$ is a positive constant depending on the finite alphabet $E$ and the system $\mathcal{S}=\left\{\phi_{e}\right\}_{e \in E}$. So, the family $\mathcal{F}(e)$ is properly placed with respect to $\left.\left(\phi_{e}^{-1}\left(x_{e}\right), \phi_{e}^{-1}(B), 2 K r\left\|\phi_{e}^{\prime}\right\|^{-1}\right)\right)$ as long as $2 K r\left\|\phi_{e}^{\prime}\right\|^{-1}<R_{*}$. Let us also remark that there exists a positive constant $K^{\prime}$ so that, for any points $x, y \in V, k \geq 1$ an integer and elements $e_{1}, \ldots, e_{k} \in E$, we have

$$
|\log | \phi_{e_{k}}^{\prime}\left|\left(\phi_{e_{k-1}} \circ \ldots \circ \phi_{e_{1}}(x)\right)-\log \right| \phi_{e_{k}}^{\prime}\left|\left(\phi_{e_{k-1}} \circ \ldots \circ \phi_{e_{1}}(y)\right)\right| \leq \tilde{D} K^{\prime} s^{k-1}|x-y| \text {, }
$$

where we recall that $\left|\phi_{e}^{\prime}\right|, e \in E$ are bounded by $s<1$. From (3.5) we see (by a classical argument involving the sum of a geometric series) that the derivatives $\phi_{\alpha}^{\prime}$ have bounded distortion independent of $\alpha \in E^{*}$; i.e., there exists a constant (denoted for simplicity also by $K$ ) s.t. $\frac{\left|\phi_{\alpha}^{\prime}(x)\right|}{\left|\phi_{\alpha}^{\prime}(y)\right|} \leq K, x, y \in V, \alpha \in E^{*}$. Proceeding now by induction we see that, given elements $\alpha, \beta \in E^{*}$ with $l:=|\alpha| \geq 2$ such that $\alpha \beta \in \mathcal{F}$ and

$$
2 K r\left\|\phi_{\alpha}^{\prime}\right\|^{-1}<R_{*}
$$

we can form similarly as above the family $\mathcal{F}\left(\alpha_{1} \ldots \alpha_{l}\right):=\mathcal{F}\left(\alpha_{1} \ldots \alpha_{l-1}\right)\left(\alpha_{l}\right)$ which is properly placed with respect to $\left(\phi_{\alpha_{l}}^{-1}\left(x_{\alpha}\right), \phi_{\alpha}^{-1}(B), 2 K r\left\|\phi_{\alpha}^{\prime}\right\|^{-1}\right)$, with $x_{\alpha}:=$ $\phi_{\alpha_{l-1}}^{-1}\left(\phi_{\alpha_{l-2}}^{-1}\left(\ldots\left(\phi_{\alpha_{1}}^{-1}\left(x_{\alpha_{1}}\right)_{\alpha_{2}}\right) \ldots\right)\right.$.

Now fix the largest integer $l \geq 1$ so that (3.6) holds for all the words $\alpha \beta \in \mathcal{F}$ starting with $\alpha$, and such that $|\alpha| \leq l$. Then, continuing (3.4), we can estimate as follows:

$$
\begin{aligned}
\Sigma(\mathcal{F}) \leq & \sum_{e_{1} \in \mathcal{F}_{1}} d^{-1}\left(z_{x}\right) \sum_{e_{2} \in \mathcal{F}\left(e_{1}\right)_{1}} d^{-1}\left(z_{x_{e_{1}}}\right) \sum_{e_{3} \in \mathcal{F}\left(e_{1} e_{2}\right)_{1}} d^{-1}\left(z_{x_{e_{1} e_{2}}}\right) \cdot \ldots \\
& \cdot \sum_{e_{l+1} \in \mathcal{F}\left(e_{1} \ldots e_{l}\right)_{1}} d^{-1}\left(z_{x_{e_{1} e_{2} \ldots e_{l}}}\right) e^{-\mathrm{P}(t)|\omega|} \\
& \left.\cdot \sum_{\omega \in \mathcal{F}\left(e_{1} e_{2} \ldots e_{l} e_{l+1}\right)} \kappa^{-1}(\omega \tau) \kappa^{-1}(\sigma(\omega \tau)) \ldots \kappa^{-1} \sigma^{|\omega|-1}(\omega \tau)\right),
\end{aligned}
$$

where we have that $\mathcal{F}\left(e_{1} e_{2} \ldots e_{l} e_{l+1}\right)=\left\{\omega \in E^{*}: e_{1} e_{2} \ldots e_{l} e_{l+1} \omega \in \mathcal{F}\right\}$, for $l \geq 1$.

Now we define a special family, which is properly placed with respect to the triple $(x, B(x, r), r)$, with $r \in\left(0, R_{*}\right)$, namely:

$\mathcal{F}_{*}(x, r):=\left\{\omega \in E^{*}: B(x, r) \cap \phi_{\omega}(J) \neq \emptyset, \phi_{\omega}(J) \subset B(x, 2 r), \phi_{\left.\omega\right|_{|\omega|-1}}(J) \not \subset B(x, 2 r)\right\}$. 
Recall also that $\left\|\phi_{e}^{\prime}\right\| \leq s<1, \forall e \in E$. Hence if $l \geq 1$ is associated to $\mathcal{F}_{*}(x, r)$ as above and if $\omega \in \mathcal{F}_{*}(x, r)$, then:

$$
\begin{aligned}
r & \leq \operatorname{diam}\left(\phi_{\left.\omega\right|_{|\omega|-1}}(J)\right) \leq \tilde{D} \operatorname{diam}(X)\left\|\phi_{\left.\omega\right|_{|\omega|-1}}^{\prime}\right\| \leq \tilde{D} \operatorname{diam}(X) \gamma^{-1}\left\|\phi_{\omega}^{\prime}\right\| \\
& \leq \tilde{D} \operatorname{diam}(X) \gamma^{-1} s^{|\omega|-l}\left\|\phi_{\left.\omega\right|_{l}}^{\prime}\right\|,
\end{aligned}
$$

where

$$
\gamma=\min _{e \in E} \inf \left\{\left|\phi_{e}^{\prime}(y)\right|: y \in J\right\} \in(0,1)
$$

But since $l \geq 1$ was taken to satisfy a maximality condition above, we infer that there exists some $\omega \in \mathcal{F}_{*}(x, r)$ such that $r\left\|\phi_{\left.\omega\right|_{l+1}}^{\prime}\right\|^{-1} \geq(2 K)^{-1} R_{*}$. Hence $\left\|\phi_{\left.\omega\right|_{l}}^{\prime}\right\| \leq 2 K\left(\gamma R_{*}\right)^{-1} r$. Combining this with (3.8), we obtain that

$$
r \leq 2 K \tilde{D} \operatorname{diam}(X) \gamma^{-2} R_{*}^{-1} s^{|\omega|-l} r
$$

or equivalently:

$$
(1 / s)^{|\omega|-l} \leq A:=2 K \tilde{D} \operatorname{diam}(X)\left(\gamma^{2} R_{*}\right)^{-1} .
$$

Hence we obtain

$$
|\omega|-l \leq \frac{\log A}{\log (1 / s)}
$$

Since $\kappa \geq 1$ and $\mathrm{P}(t)>0$, it follows from this, (3.7) and (2.1) that

$$
\Sigma\left(\mathcal{F}_{*}(x, r)\right) \leq \# E^{\frac{\log A}{\log (1 / s)}} .
$$

We have from the definition of $\mathcal{F}_{*}(x, r)$ also that

$$
4 r \geq \operatorname{diam}\left(\phi_{\omega}(J)\right) \geq \tilde{D}^{-1}\left\|\phi_{\omega}^{\prime}\right\| .
$$

Since $\mathcal{F}_{*}(x, r)$ consists of mutually incomparable words and $\pi^{-1}(B(x, r)) \subset$ $\bigcup_{\omega \in \mathcal{F}_{*}(x, r)}[\omega]$, we get from (2.3), (2.2), (3.9) and (3.10) that

$$
\begin{aligned}
\mu_{t}(B(x, r)) & \preceq \sum_{\omega \in \mathcal{F}_{*}(x, r)} e^{-\mathrm{P}(t)|\omega|}\left\|\phi_{\left.\omega\right|_{n}}^{\prime}\right\|^{t} \prod_{j=0}^{|\omega|-1} \kappa^{-1}\left(\pi\left(\sigma^{j}(\omega \tau)\right)\right) \\
& \leq(4 \tilde{D})^{t} r^{t} \sum_{\omega \in \mathcal{F}_{*}(x, r)} e^{-\mathrm{P}(t)|\omega|} \prod_{j=0}^{|\omega|-1} \kappa^{-1}\left(\pi\left(\sigma^{j}(\omega \tau)\right)\right) \\
& =(4 \tilde{D})^{t} r^{t} \Sigma\left(\mathcal{F}_{*}(x, r)\right) \\
& \leq(4 \tilde{D})^{t} \# E^{\frac{\log A}{\log (1 / s)}} r^{t} .
\end{aligned}
$$

It therefore follows from the Converse Frostman Lemma (see [1]) that $\mathrm{H}_{t}(J)>0$; consequently $\operatorname{HD}(J) \geq t$. Since $t>0$ was an arbitrary number smaller than $h_{\kappa}$, we thus conclude that

$$
\operatorname{HD}(J) \geq h_{\kappa}
$$

The proof is then complete. 


\section{UPPER BOUND}

As an upper bound for the Hausdorff dimension, we shall prove the following:

Theorem 4.1. If $\mathcal{S}=\left\{\phi_{e}\right\}_{e \in E}$ is a conformal iterated function system and $\kappa \geq 1$ is an integer satisfying $d(x) \geq \kappa$ for all $x \in J$, then $\operatorname{HD}(J) \leq h_{\kappa}$.

Proof. Fix $t>h_{\kappa}$. Then $\mathrm{P}(t)<0$ and therefore

$$
\sum_{|\omega|=n}\left\|\phi_{\omega}^{\prime}\right\|^{t} e^{-\kappa n} \leq e^{\frac{1}{2} \mathrm{P}(t) n}
$$

for all $n \geq 1$ large enough, say $n \geq n_{0}$. For every $\omega \in E^{n}$ consider the smallest closed ball $B_{\omega}$ containing $\phi_{\omega}(X)$. Then

$$
\operatorname{diam}\left(B_{\omega}\right) \leq 2 \operatorname{diam}\left(\phi_{\omega}(X)\right) \leq 2 \tilde{D} \operatorname{diam}(X)\left\|\phi_{\omega}^{\prime}\right\| .
$$

Since $\left\{B_{\omega}\right\}_{\omega \in E^{n}}$ is a cover of the limit set $J$ by closed balls, by virtue of the $5 r$ Covering Theorem (see [1] and compare [3], where $5 r$ is improved to $4 r$ and, more importantly, totally bounded metric spaces are replaced by all metric spaces) there exists a set $I_{1} \subset E^{n}$ with the following properties:

(a) $B_{\omega} \cap B_{\tau}=\emptyset$ for all $\omega, \tau \in I_{1}$ with $\omega \neq \tau$;

(b) $\bigcup_{\omega \in I_{1}} 5 B_{\omega} \supset J$.

Suppose now by induction that the sets $I_{1}, I_{2}, \ldots, I_{l}, 1 \leq l<\kappa^{n}$ have been defined with the following properties:

(c) $I_{i} \cap I_{j}=\emptyset$ for all $1 \leq i<j \leq l$,

(d) $\forall(1 \leq j \leq l) \forall\left(\omega, \tau \in I_{j}\right) \omega \neq \tau \Rightarrow B_{\omega} \cap B_{\tau}=\emptyset$,

(e) $\forall(1 \leq j \leq l) \bigcup_{\omega \in I_{j}} 5 B_{\omega} \supset J$.

Because of (c) and (d), each point of $J$ belongs to at most $l$ elements of the family $\left\{B_{\omega}: \omega \in I_{1} \cup \ldots \cup I_{l}\right\}$. But, as $d \geq \kappa$, each element of $J$ belongs to at least $\kappa^{n}>l$ elements of the family $\left\{\phi_{\omega}(J):|\omega|=n\right\}$ and, thus, to at least $\kappa^{n}>l$ elements of the family $\left\{\phi_{\omega}(X):|\omega|=n\right\}$, and eventually to at least $\kappa^{n}>l$ elements of the family $\left\{B_{\omega}:|\omega|=n\right\}$. Thus, the family $\left\{B_{\omega}: \omega \in E^{n} \backslash\left(I_{1} \cup \ldots \cup I_{l}\right)\right\}$ covers $J$, and it therefore follows from the $5 r$-Covering Theorem (see [1]) that one can find a set $I_{l+1} \subset E^{n} \backslash\left(I_{1} \cup \ldots \cup I_{l}\right)$ such that

(f) if $\omega, \tau \in I_{l+1}$ and $\omega \neq \tau$, then $B_{\omega} \cap B_{\tau}=\emptyset$;

(g) $\bigcup_{\omega \in I_{l+1}} 5 B_{\omega} \supset J$.

So, we have constructed by induction a family of sets $I_{1}, I_{2}, \ldots, I_{\kappa^{n}} \subset E^{n}$ such that the conditions (c), (d), and (e) hold with $l=\kappa^{n}$.

Now choose $1 \leq j \leq \kappa^{n}$ so that the sum $\sum_{\omega \in I_{j}} \operatorname{diam}^{t}\left(B_{\omega}\right)$ is the smallest. Then by (4.2), (4.1) and (c), (d), (e), we get that

$$
\begin{aligned}
\sum_{\omega \in I_{j}} \operatorname{diam}^{t}\left(5 B_{\omega}\right) & =5^{t} \sum_{\omega \in I_{j}} \operatorname{diam}^{t}\left(B_{\omega}\right) \leq \frac{5^{t}}{\kappa^{n}} \sum_{i=1}^{\kappa^{n}} \sum_{\omega \in I_{i}} \operatorname{diam}^{t}\left(B_{\omega}\right) \\
& \leq 5^{t} \kappa^{-n} \sum_{|\omega|=n} \operatorname{diam}^{t}\left(B_{\omega}\right) \leq(10 \tilde{D} \operatorname{diam}(X))^{t} \sum_{|\omega|=n}\left\|\phi_{\omega}^{\prime}\right\|^{t} e^{-\log \kappa n} \\
& \leq(10 \tilde{D} \operatorname{diam}(X))^{t} e^{\frac{1}{2} \mathrm{P}(t) n} .
\end{aligned}
$$

Because of (e) and since $\mathrm{P}(t)<0$, we thus conclude that $\mathrm{H}_{t}(J)=0$, so $\mathrm{HD}(J) \leq t$. By the arbitrariness of $t>h_{\kappa}$, this yields $\operatorname{HD}(J) \leq h_{\kappa}$. We are done. 
As a consequence of Theorem 3.1 and Theorem 4.1 we get the following.

Corollary 4.2. Suppose that $\mathcal{S}=\left\{\phi_{e}\right\}_{e \in E}$ is a conformal iterated function system and let $D:=\max \left\{d(x): x \in J_{\mathcal{S}}\right\}$. Then $\operatorname{HD}\left(J_{\mathcal{S}}\right)=h_{D}$ if and only if $d(x)=D$ for all $x \in J_{S}$.

Proof. If $d(x)=D$ for all $x \in J_{S}$, then the equality $\operatorname{HD}\left(J_{\mathcal{S}}\right)=h_{D}$ is a direct consequence of Theorem 3.1 and Theorem 4.1 .

In order to prove the converse, suppose that $h:=\operatorname{HD}\left(J_{\mathcal{S}}\right)=h_{D}$. By way of a contradiction suppose that there exists $z \in J$ such that $d(z) \leq D-1$. Since the alphabet $E$ is finite, there thus exists an open neighborhood $V$ of $z$ such that $d(x) \leq D-1$ for all $x \in V$. Fix a nonempty open set $U \subset J$ such that $\bar{U} \subset V$. There then exists a Lipschitz function $\hat{\kappa}: J \rightarrow[1,+\infty)$ such that $\hat{\kappa}(x)=D-1$ for all $x \in \bar{U}$ and $\hat{\kappa}(x)=D$ for all $x \in J \backslash V$. In particular, $d(y) \leq \hat{\kappa}$ for all $y \in J$, and it therefore follows from Theorem 3.1 that $h_{D}=h \geq h_{\kappa}$; recall that $\kappa=\hat{\kappa} \circ \pi$. But we also have

$$
\kappa \leq D \text { on } E^{\infty}
$$

and thus $h_{D} \leq h_{\kappa}$. Hence,

$$
h_{\kappa}=h_{D} .
$$

Let $\tilde{\mu}_{D}$ be the unique equilibrium (Gibbs) state on $E^{\infty}$ of the potential $h_{d} \psi-\log D$. Since $\mathrm{P}\left(h_{D} \psi-\log D\right)=0$, we have

$$
\int_{E^{\infty}}\left(h_{D} \psi-\log D\right) d \tilde{\mu}_{D}+\mathrm{h}_{\tilde{\mu}_{D}}(\sigma)=0,
$$

where $\mathrm{h}_{\tilde{\mu}_{D}}(\sigma)$ is the Kolmogorov-Sinai metric entropy of the dynamical system $\sigma: E^{\infty} \rightarrow E^{\infty}$ with respect to the $\sigma$-invariant measure $\tilde{\mu}_{D}$. By virtue of the Variational Principle, we also have

$$
\begin{aligned}
\int_{E^{\infty}}\left(h_{D} \psi-\log \kappa\right) d \tilde{\mu}_{D}+\mathrm{h}_{\tilde{\mu}_{D}}(\sigma) & =\int_{E^{\infty}}\left(h_{\kappa} \psi-\log D\right) d \tilde{\mu}_{D}+\mathrm{h}_{\tilde{\mu}_{D}}(\sigma) \leq \mathrm{P}\left(h_{\kappa} \psi-\log \kappa\right) \\
& =0 .
\end{aligned}
$$

This, combined with (4.5), implies that

$$
\int_{E^{\infty}}(\log D-\log \kappa) d \tilde{\mu}_{D} \leq 0 .
$$

Since the function $\log D-\log \kappa$ is continuous and since the equilibrium state $\tilde{\mu}_{D}$ (as a Gibbs state of a Hölder continuous function) is positive on nonempty open subsets of $E^{\infty}$, it follows from (4.6) and (4.3) that $\log \kappa=\log D$ on $E^{\infty}$. So, $\hat{\kappa}=D$ on $J$, and this contradiction finishes the proof.

Examples where the above estimates apply can be formed by taking a finite number of conformal contractions $\phi_{e}, e \in E$ and checking the number of sets $\phi_{e}(V)$ intersecting each other in a certain domain $W$, and seeing whether we may have points of $J$ in $W$ or not. For the pressure on $E^{\infty}$, one can take canonical spanning sets for the shift map in $E^{\infty}$, consider the behavior of $\kappa$ on such sets and then estimate the zero $h_{\kappa}$ of the pressure function. Or one may recall the fact that the entropy of $\sigma$ on $E^{\infty}$ is equal to $\log \operatorname{Card}(E)$ and then use bounds on $\kappa$ in order to estimate $h_{\kappa}$. 


\section{REFERENCES}

[1] P. Mattila, Geometry of sets and measures in Euclidean spaces. Fractals and rectifiability. Cambridge Studies in Adv. Math., 44, Cambridge Univ. Press, 1995. MR1333890 (96h:28006)

[2] D. Mauldin and M. Urbański, Graph Directed Markov Systems: Geometry and Dynamics of Limit Sets, Cambridge Univ. Press, 2003. MR2003772 (2006e:37036)

[3] R. D. Mauldin, T. Szarek, and M. Urbański, Graph directed Markov systems on Hilbert spaces, Math. Proc. Cambridge Phil. Soc. 147 (2009), 455-488. MR2525938 (2010i:28015)

[4] E. Mihailescu, Unstable directions and fractal dimension for skew products with overlaps in fibers, arXiv:0911.2345, Math. Zeitschrift, DOI 10.1007/s00209-010-0761-y

[5] E. Mihailescu and M. Urbanski, Inverse pressure estimates and the independence of stable dimension for non-invertible maps, Canadian J. Math., 60, no. 3 (2008), 658-684. MR2414959 (2009c:37028)

[6] E. Mihailescu and M. Urbański, Estimates for the stable dimension for holomorphic maps, Houston J. Math. 31 (2005), 367-389. MR2132842 (2005m:37108)

[7] K. Simon and B. Solomyak, Hausdorff dimension for horseshoes in $\mathbb{R}^{3}$, Ergodic Th. and Dynam. Sys. 19 (1999), 1343-1363. MR1721625 (2001k:37041)

[8] B. Solomyak, Non-linear iterated function systems with overlaps, Periodica Math. Hungarica 37 (1-3) (1998), 127-141. MR1719444 (2001b:28014)

Institute of Mathematics "Simion Stoilow" of the Romanian Academy, P. O. Box 1-764, RO 014700, Bucharest, Romania

E-mail address: Eugen.Mihailescu@imar.ro

$U R L$ : www.imar.ro/ mihailes

Department of Mathematics, University of North Texas, P.O. Box 311430, Denton, TEXas 76203-1430

E-mail address: urbanski@unt.edu

$U R L:$ www.math.unt.edu/ urbanski 